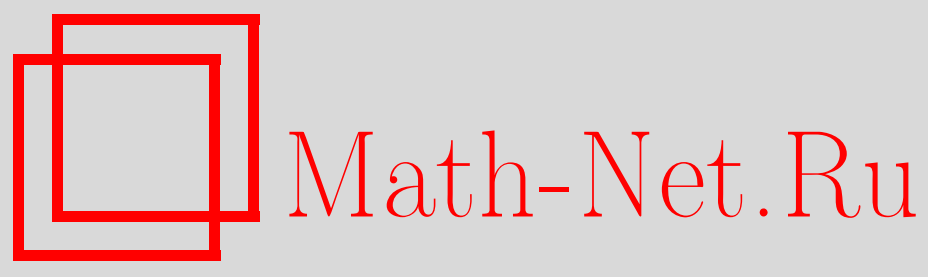

Х. Д. Икрамов, Л. Эльзнер, О матрицах, допускающих унитарное приведение к ленточному виду, Матем. заметки, 1998, том 64, выпуск 6, 871-880

DOI: https://doi.org/10.4213/mzm1466

Использование Общероссийского математического портала Math-Net.Ru подразумевает, что вы прочитали и согласны с пользовательским соглашением http://www . mathnet.ru/rus/agreement

Параметры загрузки:

IP : 3.85 .5 .30

26 апреля 2023 г., $15: 27: 08$

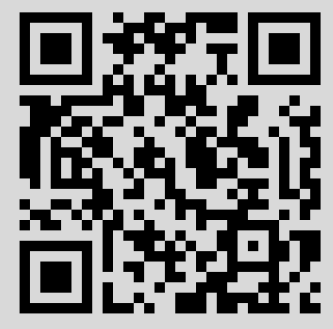




\title{
О МАТРИЦАХ, ДОПУСКАЮЩИХ УНИТАРНОЕ ПРИВЕДЕНИЕ К ЛЕНТОЧНОМУ ВИДУ
}

\section{Х. Д. Икрамов, Л. Эльзнер}

\begin{abstract}
В статье доказано, что всякое малоранговое возмущение эрмитовой матрицы унитарно приводимо к ленточной форме. Кроме того, если нормальная матрица унитарно приводима к ленточной форме, то унитарно приводимо и всякое ее одноранговое возмущение.

Библиография: 11 названий.
\end{abstract}

1. Введение. Хорошо известно, что всякую эрмитову матрицу можно привести к трехдиагональному виду с помощюю конечной последовательности элементарных унитарных подобий. В качестве трансформирующих матриц этих подобий пригодны, например, отражения или комплексные аналоги двумерных вращений. И в том, и в другом случае процесс приведения к трехдиагональной форме использует лишь арифметические операции и извлечения квадратных корней из положительных чисел. Тем самым, унитарное приведение эрмитовой матрицы к трехдиагональному виду реализуемо в квадратичных радикалах.

В недавней публикации [1] установлено, что всякая унитарная матрища унитарно приводима, опять-таки в квадратичных радикалах, к пятидиагональному виду. Этот результат послужил стимулом проведенного в [2] исследования на тему, возможно ли унитарное приведение (в квадратичных радикалах) к нетривиальной ленточной форме для произвольной нормальной матрицы $A$. Ответ отрищателен в общем случае и положителен, если $A$ вместе с сопряженной матрищей $A^{*}$ удовлетворяет алгебраическому соотношению

$$
f\left(A, A^{*}\right)=0,
$$

где $f(x, y)$ - многочлен не слишком высокой степени.

Подчеркнем условие конечности, входящее в наше понимание приводимости. Если отказаться от этого условия, то всякая нормальная матрица становится унитарно приводимой даже к диагональному виду. Унитарная приводимость в таком смысле есть, как известно, характеристическое свойство нормальных матриц.

Среди симметричных ленточных форм следующей по простоте за диагональной является трехдиагональная форма. По отношению к ней справедлива

Теорема 1. Для всякого $n \geqslant 5$ существуют комплексные $(n \times n)$-матрицы, которые никаким унитарным подобием не могут быть преобразованы к трехдиагональному виду.

Этот результат для $n \geqslant 6$ был установлен в [3], [4]. В [5] показано, что, несколько усовершенствовав доказательство Штурмфельса из [4], можно распространить его и на случай $n=5$. 
Заметим, что матрицы, не приводимые (в расширенном толковании) к трехдиагональному виду, образуют при $n \geqslant 5$ всюду плотное подмножество пространства $(n \times n)$ матриц. Аналогичное утверждение (при соответствующих ограничениях на $n$ ) имеет место для ленточной формы любой фиксированной ширины $p$. Тем больший интерес представляют те сравнительно немногочисленные матрицы, которые допускают унитарное приведение к ленточному виду, а тем более матрицы, чье приведение возможно осуществить в квадратичных радикалах.

Цель работы - указать некоторые классы таких матриц. Мы устанавливаем, что всякая матрица с косоэрмитовой компонентой малого ранга унитарно приводима к ленточной форме, и такое приведение можно реализовать в квадратичных радикалах (теоремы 8 и 9). В теореме 10 показано, что одноранговое возмущение нормальной матрищы, допускающей унитарноеприведение к ленточному виду, обладает аналогичньм свойством приводимости.

Средством доказательства наших результатов является обобщенный процесс Ланцоша, в п. 2 дается его описание и найденные в [2] свойства, важные для дальнейшего изложения. Роль линейных зависимостей в обобщенной степенной последовательности матриц обсуждается в п. 3. Наконец, в п. 4 формулируются и доказьваются главные результаты.

2. Обобщенный процесс Ланцоша. Пусть задана $(n \times n)$-матрица $A$ и фиксирован ненулевой начальный вектор $v$. Обобщенной последовательностью Крылова назовем последовательность векторов

$$
v, A v, A^{*} v, A^{2} v, A^{*} A v, A A^{*} v, A^{* 2} v, A^{3} v, \ldots
$$

Удобно рассматривать последовательность (2) как состоящую из сегментов длины соответственно $1,2,4,8,16, \ldots$. Сегмент с номером $k$, мы назьваем его $k$-м слоем, можно описать как совокупность векторов вида $u=W_{k}\left(A, A^{*}\right) v$, где $W_{k}(s, t)$ пробегает множество слов степени $k$ от двух некоммутирующих переменных $s$ и $t$. Порядок слов внутри слоя может быть произвольным. Символ $W_{0}(s, t)$ обозначает единицу.

Наряду с векторной последовательностью (2) (или даже вместо нее) будем использовать матричную последовательность

$$
I, A, A^{*}, A^{2}, A^{*} A, A A^{*}, A^{* 2}, A^{3}, A^{2} A^{*}, A A^{*} A, \ldots, A^{* 3}, \ldots
$$

Мы назьваем ее обобщенной степенной последовательностью. Понятие слоя определяется для (3) по аналогии с (2).

Суть обобщенного процесса Ланцоша, формальное описание которого дается ниже, состоит в ортогонализации последовательности (2). Пусть $v_{1}, v_{2}, v_{3}, \ldots$ - получаемые при этом ненулевые ортогональные векторы. Положим

$$
\mathscr{K}_{m}=\operatorname{span}\left\{v_{1}, \ldots, v_{m}\right\}
$$

Обозначим через $\operatorname{orth}_{\mathscr{K}} w$ ортогональную составляющую в разложении вектора $w$ относительно линейного подпространства $\mathscr{K}$.

Обобщенный процесс Ланцоша состоит в следующем.

1. Выбрать ненулевой вектор $v$ и положить $v_{1}=v$. 
2. Пусть уже построены ненулевые векторы $v_{1}, \ldots, v_{m}$, составляющие ортогональньй базис линейной оболочки первых $k_{m}$ векторов $u_{1}, \ldots, u_{k_{m}}$ последовательности (2). Если перпендикуляр orth $\mathscr{K}_{m} u_{k_{m}+1}$ не равен 0 , то принять его в качестве $v_{m+1}$ и положить $k_{m+1}=k_{m}+1$. В противном случае построить вектор orth $\mathscr{K}_{m} u_{k_{m}+2}$. Если он ненулевой, принять его в качестве $v_{m+1}$; положить $k_{m+1}=k_{m}+2$, и т.д.

ЗАмЕЧАнИЕ 1 . Если все векторы $k$-го слоя последовательности (2) дают нулевые перпендикуляры на текущее подпространство $\mathscr{K}_{m}$, то $\mathscr{K}_{m}$ есть общее инвариантное подпространство матриц $A$ и $A^{*}$. Процесс Ланцоша в этом случае завершается досрочно.

В дальнейшем мы придерживаемся терминологии и обозначений, введенных в [2]. Обобщенным подпространством Крылова (с номером $m$ ) назьвается подпространство

$$
L_{m}(A, v)=\operatorname{span}\left\{W\left(A, A^{*}\right) v: \operatorname{deg} W \leqslant m\right\} .
$$

Его размерность обозначается через $l_{m}$. Число $w_{m}=l_{m}-l_{m-1}(m \geqslant 1)$ назьвается шириной $m$-го слоя; мы полагаем $w_{0}=1$. Аналогичные определения применяются $\mathrm{k}$ последовательности (3). Ясно, что для каждой из этих последовательностей

$$
w_{m} \leqslant 2^{m}
$$

Укажем теперь связь между процессом Ланцоша и задачей об унитарном приведении к ленточной форме. Предположим, что линейная оболочка последовательности (2) совпадает с пространством $\mathbb{C}^{n}$. Тогда процесс Ланцоша генерирует ортогональньй базис $v_{1}, \ldots, v_{n}$; нормируя векторы $v_{i}$, можем считать базис даже ортонормированным. Интерпретируя исходную матрицу $A$ как оператор $\mathscr{A}$ на унитарном пространстве $\mathbb{C}^{n}$, построим матрицу $B$ этого оператора в базисе $v_{1}, \ldots, v_{n}$. Структурные особенности матрицы $B$ можно описать следуюшим образом. Пусть $s$ - номер слоя последовательности (2), породившего вектор $v_{i}$. В таком случае в $i$-м столбце матрицы $B$ отличными от 0 могут быть лишь элементы, соответствующие векторам $v_{j}$, порожденньг $(s-1)$, $s$ - и $(s+1)$-м слоями. Аналогичное описание применимо к строкам $B$. Обозначая через $N_{i}\left(M_{i}\right)$ число ненулевых элементов в $i$-м столбце ( $i$-й строке) матрицы $B$, получаем неравенства

$$
N_{i} \leqslant w_{s-1}+w_{s}+w_{s+1}, \quad M_{i} \leqslant w_{s-1}+w_{s}+w_{s+1} .
$$

ЗАмЕчАниЕ 2. Если для всех $s$ имеет место равномерная оценка

$$
w_{s} \leqslant b
$$

то $B$ является ленточной матрицей с шириной ленты, не превосходящей $4 b-1$. Так как проведение обобщенного процесса Ланщоша требует лиш арифметических операций и квадратных корней, матрица $A$ в ситуации (5) оказывается унитарно приводимой к ленточному виду.

Важный случай, когда неравенство (5) действительно вьполняется, приведен в [2]. Предположим, что $A$ - нормальная матрица и в последовательности (3) матрищы, принадлежащие слоям $0,1, \ldots, m_{0}-1$, линейно независимы в совокупности. Пусть, далее, присоединение матрищ из слоя $m_{0}$ превращает эту матричную систему в линейно зависимую. Иньми словами, $A$ и $A^{*}$ удовлетворяют уравнению вида $(1)$, где $f$ - многочлен степени $m_{0}$. Тогда $w_{s} \leqslant w_{m_{0}}$ для всех $s$. 
Приведенному описанию удовлетворяют эрмитовы, косоэрмитовы и унитарные матрицы, а также нормальные матрищы, спектр которых сосредоточен на любой алгебраической кривой невысокой степени.

Если (в соответствии с замечанием 1) процесс Ланцоша порождает лиш базис нетривиального инвариантного подпространства матриц $A$ и $A^{*}$, то при соответствующем изменении базиса в $\mathbb{C}^{n}$ мы получим из $A$ блочнодиагональную матрицу, к верхнему диагональному блоку которой применимы изложенные выше соображения. Полноеприведение $A$ в этом случае требует повторного обращения к процедуре Ланцоша с другим начальньц вектором.

3. Линейные зависимости в обобщенной степенной последовательности. В общем случае наличие линейньх зависимостей в обобщенной степенной последовательности (3) влечет уменьшение ширины слоев, но не ее стабилизацию. Предположим, например, что матрица $A$ в $(3)$ нормальная. Нормальность, описываемая равенством

$$
A^{*} A=A A^{*},
$$

есть линейное соотношение во втором слое последовательности (3). Однако, при отсутствии других линейных зависимостей числа $w_{m}$ сохраняют медленньй рост: $w_{m}=m+1$, хотя скорость его не сравнима с максимальным ростом, допускаемым оценкой (4). Вместе с тем, как указано в п. 2, добавочные линейные соотношения стабилизируют последовательность $\left\{w_{m}\right\}$. Так, для эрмитовой матрищы $A$ имеем $w_{m} \leqslant 1$ для всех $m$; если $A$ унитарная, то $w_{m} \leqslant 2$ при $m \geqslant 1$, и т.д.

Приведем еще один пример стабилизации чисел $w_{m}$, относящийся на этот раз к $а н о p$ мальным матрицам (т.е. матрицам, не являющимся нормальными). Пусть $A$ - косой проектор, т.е.

$$
A^{2}=A, \quad A^{*} A \neq A A^{*} .
$$

Из первого соотношения (7) вьводим $A^{* 2}=A^{*}$. Отсюда легко следует, что каждьй слой последовательности (3) может добавить к предыдущим лишь две новые матрицы, а именно: $\left(A A^{*}\right)^{k}$ и $\left(A^{*} A\right)^{k}$, если номер слоя $m$ есть четное число $(m=2 k)$, и $A\left(A^{*} A\right)^{k},\left(A^{*} A\right)^{k} A^{*}$ при $m=2 k+1$. Таким образом, $w_{m} \leqslant 2$ при $m \geqslant 1$.

Почти не меняя рассуждений, можно показать, что аналогичная стабилизация имеет место для всякой матрицы $A$ с квадратичным минимальньг многочленом. К числу таких матрищ, помимо проекторов, относятся инволюции, а также нильпотенты и унипотенты индекса 2.

ЗАмЕчАнИЕ 3. Если в рассмотренном примере применение процесса Ланцоша к последовательности (2) порождает ортогональньй базис в $\mathbb{C}^{n}$, то матрица $A$ оказьвается унитарно подобной ленточной матрице с шириной ленты 7 (см. замечание 2). И здесь уместно подчеркнуть роль требования конечности в процессе приведения. Если допустить произвольные унитарные подобия, то матрица с квадратичным минимальным многочленом приводима даже к блочнодиагональному виду с диагональньми блоками порядков 1 и 2 (см. [6, п. 2]).

ЗАМЕЧАНИЕ 4. Для того чтобы из обобщенной крыловской последовательности (2) можно было выделить базис для $\mathbb{C}^{n}$, необходимо, прежде всего, наличие достаточного числа линейно независимых матриц в последовательности (3). Последнее может вызьвать сомнения в случае матрицы с квадратичным минимальным многочленом: достаточно взглянуть на первое соотношение (7). Тем не менее, имеет место следующий факт [7]. 
Теорема 2. Пусть $A$ и $B-(n \times n)$-матрииы над произвольным полем $F$, имеющие квадратичные минимальные многочлены. Тогда для размерности алгебры $\mathscr{B}$, порождаемой над $F$ матрицами $I, A$ и $B$, справедливы оченки

$$
\operatorname{dim} \mathscr{B} \leqslant \begin{cases}2 n-1, & \text { если } n \text { нечетно } \\ 2 n, & \text { если } n \text { четно. }\end{cases}
$$

В интересующей нас ситуации теорему 2 следует применять к паре $A, A^{*}$.

Для нормальной матрицы $A$ всякое линейное соотношение

$$
\alpha_{1} A^{s_{1}} A^{* t_{1}}+\alpha_{2} A^{s_{2}} A^{* t_{2}}+\cdots+\alpha_{l} A^{s_{l}} A^{* t_{l}}=0
$$

в последовательности (3) влечет аналогичное скалярное соотношение

$$
\alpha_{1} \lambda^{s_{1}} \bar{\lambda}^{t_{1}}+\alpha_{2} \lambda^{s_{2}} \bar{\lambda}^{t_{2}}+\cdots+\alpha_{l} \lambda^{s_{l}} \bar{\lambda}^{t_{l}}=0,
$$

которому должно удовлетворять каждое собственное значение $\lambda$ матрицы $A$. В случае анормальных матриц аналогичное утверждение, вообще говоря, неверно. Однако, справедлив следующий его частичньй эквивалент [8].

ТЕОРема 3. Пусть задан многочлен

$$
f(x, y)=\sum_{\mu=0}^{m} \sum_{\nu=0}^{l} a_{\mu \nu} x^{\mu} y^{\nu} .
$$

Рассмотрим определяемое им матричное уравнение относительно $(n \times n)$-матричй A:

$$
\sum_{\mu=0}^{m} \sum_{\nu=0}^{l} a_{\mu \nu} A^{\mu} A^{* \nu}=0 .
$$

Если $A$ - решение уравнения (8), то для всякого собственного значения $\lambda$ матрииы $А$ выполняется равенство $f(\lambda, \bar{\lambda})=0$.

Так, уравнению

$$
A^{2}-A^{* 2}=0
$$

удовлетворяют, в частности, все инволюции, собственные числа которых, как известно, могут принимать лиш значения 1 и -1 . Этот пример показьвает, что уравнение вида (8) может допускать анормальные решения.

Вместе с тем, некоторым уравнениям типа (8) удовлетворяют лишь нормальные матрицы. Простейшими иллюстрациями являются определения эрмитовых $\left(A=A^{*}\right)$ и унитарных $\left(A A^{*}=I\right)$ матриц. Существуют и менее избитые примеры. Напомним в связи с этим два результата из [9].

Теорема 4. Пусть $A$ - комплексная $(n \times n)$-матрица с собственнымии значениями $\lambda_{1}, \ldots, \lambda_{n}$. Предположим, что для некоторых комплексных чисел $a_{1}, a_{2}, b_{1}, b_{2}$, $c_{1}, c_{2}$, әде $c_{1}+c_{2} \neq 0$, спектр матрици

$$
B=a_{1} A+a_{2} A^{*}+b_{1} A^{2}+b_{2} A^{* 2}+c_{1} A^{*} A+c_{2} A A^{*}
$$

состоит из чисел

$$
\mu_{j}=a_{1} \lambda_{j}+a_{2} \bar{\lambda}_{j}+b_{1} \lambda_{j}^{2}+b_{2} \bar{\lambda}_{j}^{2}+\left(c_{1}+c_{2}\right)\left|\lambda_{j}\right|^{2}, \quad j=1, \ldots, n .
$$

В таком случае $A$ является нормальной матрицей. 
Теорема 5. Допустим, что в условиях теоремы 4 требование $c_{1}+c_{2} \neq 0$ заменено соотношениями

$$
a_{2}=\bar{a}_{1}, \quad b_{2}=\bar{b}_{1}, \quad c_{1}, c_{2} \in \mathbb{R}, \quad c_{1}^{2}+c_{2}^{2} \neq 0 .
$$

Тогда матрииа $A$ по-прежнему нормальна.

На основе каждого из этих утверждений можно сформулировать достаточный критерий нормальности. Для определенности, используем теорему 4.

ТЕОРема 6. Пусть задан квадратичный многочлен

$$
f(x, y)=a_{00}+a_{10} x+a_{01} y+a_{20} x^{2}+a_{11} x y+a_{02} y^{2},
$$

где $a_{11} \neq 0$. Тогда всякая матрича $A$, удовлетворяющая уравнению

$$
f\left(A, A^{*}\right)=a_{00} I+a_{10} A+a_{01} A^{*}+a_{20} A^{2}+a_{11} A A^{*}+a_{02} A^{* 2}=0,
$$

является нормальной.

ДокАЗАтЕЛЬСтво. Положим $B=f\left(A, A^{*}\right)$. По условию $B=0$. Согласно теореме $3 f(\lambda, \bar{\lambda})=0$ для всякого собственного значения $\lambda$ матрицы $A$. Поскольку $a_{11} \neq 0$, условия теоремы 4 выполнены для $A$ и $B$, и матрища $A$ нормальная.

ЗАмЕчаниЕ 5. Вместо уравнения $f\left(A, A^{*}\right)=0$ в теореме 6 можно рассматривать уравнение $f\left(A^{*}, A\right)=0$. Эти два уравнения, по-существу, различаются лишш тем, что в одно из них входит первое из произведений $A A^{*}$ и $A^{*} A$, а в другое-второе.

ЗАмЕчАниЕ 6. Вещественная алгебраическая кривая

$$
a_{11} x^{2}+2 a_{12} x y+a_{22} y^{2}=1
$$

может быть записана комплексным уравнением

$$
c z^{2}+\overline{c z}^{2}+2 d z \bar{z}=4
$$

где $c=a_{11}-a_{22}-2 a_{12} i, d=a_{11}+a_{22}$. В [2] посредством соотношений

$$
A A^{*}=A^{*} A, \quad c A^{2}+\bar{c} A^{* 2}+2 d A A^{*}=4 I
$$

был введен класс Г нормальных матриц со спектром, сосредоточенньпм на кривой (10). Как следует из теоремы 6, при $d \neq 0$ первое условие (11) избыточно: нормальность матриц $A \in$ Г обеспечивается вторым соотношением. В то же время, пример (9) показывает необходимость первого условия в случае $d=0$.

Предположим, что одночлены

$$
A^{s_{1}} A^{* t_{1}} A^{s_{2}} A^{* t_{2}} \cdots A^{s_{l}} A^{* t_{l}}, \quad s_{1}+t_{1}+\cdots+s_{l}+t_{l}=m,
$$

входящие в $m$-й слой последовательности (3), удовлетворяют линейному соотношению над $L_{m-1}$ (т.е. некоторая нетривиальная их линейная комбинация принадлежит подпространству $\left.L_{m-1}\right)$. Назовем это соотношение симметричным, если выполнены условия:

а) для каждого одночлена (12), входящего в соотношение с ненулевым коэффициентом $\beta$, оно содержит и одночлен

$$
A^{t_{l}} A^{* s_{l}} \cdots A^{t_{1}} A^{* s_{1}}
$$

также с ненулевым коэффициентом $\gamma$;

б) $|\beta|=|\gamma|$. 
Если хотя бы одно из этих условий нарушено, соотношение называется несимметричным. Уравнение (6) может служить примером симметричного соотношения во втором слое. Определение проектора (более общо, любой матрицы с квадратичным минимальным многочленом), переписанное как $A^{2}=0\left(\bmod L_{1}\right)$, есть пример несимметричного соотношения.

ТЕОРема 7. Пусть имеем несимметричное линейное соотношение в последовательности (3). Тогда применение к нему операчии сопряжения порохдает новое линейное соотношение, линейно независимое с исходным.

ДокАЗАТЕЛЬСтво. Утверждение теоремы очевидно, если существует пара одночленов (12) и (13), из которых один входит в заданное соотношение, а другой - нет. Предположим, что одночлены $(12),(13)$ входят в соотношение с ненулевыми коэффициентами $\beta$ и $\gamma$, однако $|\beta| \neq|\gamma|$. В новое соотношение те же одночлены войдут с коэффициентами $\bar{\gamma}$ и $\bar{\beta}$. Остается заметить, что

$$
\left|\begin{array}{ll}
\beta & \frac{\gamma}{\gamma}
\end{array}\right|=|\beta|^{2}-|\gamma|^{2} \neq 0
$$

В качестве примера рассмотрим соотношение $\left[A A^{*} A, A\right]=0$, или

$$
A^{2} A^{*} A-A A^{*} A^{2}=0
$$

Для невырожденной матрицы $A$ уравнение (14) эквивалентно условию нормальности (6). Среди его вырожденных решений имеются и анормальные. Для этого (вырожденного) случая уравнение было введено в [10] как одно из возможных описаний класса матриц $A$, для которых $A^{*}$ и $A^{+}$(псевдообратная матрица Мура-Пенроуза) перестановочны. Поскольку соотношение (14), очевидньм образом, несимметрично, сопряженное соотношение $A^{*} A A^{* 2}-A^{* 2} A A^{*}=0$ с ним линейно независимо.

4. Матрицы, унитарно приводимые к ленточной форме. Пусть

$$
A=B+i C, \quad B=\frac{1}{2}\left(A+A^{*}\right), \quad C=\frac{1}{2 i}\left(A-A^{*}\right),
$$

- теплищево разложение матрицы $A$. Последовательность

$$
I, B, C, B^{2}, B C, C B, C^{2}, B^{3}, B^{2} C, B C B, C B^{2}, C B^{2}, \ldots, C^{3}, \ldots
$$

эквивалентна последовательности (3) в том смысле, что, будучи разделена на слои, генерирует ту же последовательность $\left\{L_{m}\right\}$ обобщенных крыловских подпространств. Последовательность (16) оказьвается более удобной для доказательства теорем 8 и 9 .

Теорема 8. Пусть для матрицы $A$ из (15) $\operatorname{rank} C=1 . B$ таком случае $A$ может быть унитарно преобразована в ленточную матрицу с иириной ленты, не превосходящей 7, посредством конечного прочесса, использующего лииь арифметические операчии и квадратные корни. 
ДокАЗАТЕЛЬСТво. Для начального вектора $v$ рассмотрим последовательность

$$
v, B v, C v, B^{2} v, B C v, C B v, C^{2} v, B^{3} v, B^{2} C v, \ldots,
$$

эквивалентную последовательности (2). Из условия теоремы легко вытекает, что слои последовательности (17) имеют ширину $\leqslant 2$. Если процесс Ланщоша, примененньй к $(17)$, порождает ортогональный базис в $\mathbb{C}^{n}$, то переход к этому базису дает нужную ленточную матрицу, унитарно подобную матрице $A$. Иначе, мы имеем лишь ортогональный базис общего для $A$ и $A^{*}$ инвариантного подпространства $\mathscr{L}$. Выбрав ненулевой вектор в ортогональном дополнении $\mathscr{L}^{\perp}$, проведем процесс Ланцоша еще раз. Не исключено, что может потребоваться и неоднократное повторение. Однако, в конечном счете, ортогональный базис для $\mathbb{C}^{n}$ будет построен, а вместе с ним и ленточная форма требуемой ширины для $A$. Отличие от первого случая состоит в том, что эта форма будет прямой суммой не менее чем двух ленточных матриц.

ЗАмЕчАниЕ 7. Пусть $t$ - ненулевой вектор из образа матрицы $C$. Найти такой вектор несложно; например, годится любой ненулевой столбец самой матрищы $C$. Полагая в (17) $v=t$, мы без труда убедимся, что эта последовательность эквивалентна (снова с точки зрения генерируемых крыловских подпространств) обычной степенной последовательности $t, B t, B^{2} t, B^{3} t, \ldots$ Если последняя порождает все пространство $\mathbb{C}^{n}$, то, ортонормируя ее, приходим даже к трехдиагональной форме для матрицы $A$. Это один из результатов статьи [3]. Теорема 8 отличается от него тем, что позволяет выбор произвольного начального вектора. Как следствие, ширина ленты возрастает с 3 до 7.

Несколько усложняя рассуждения, можно установить следующий более общий результат.

Теорема 9. Пусть для матрииы $A$ из (15) $\operatorname{rank} C=k$, әде $k<(n-2) / 2 . B$ таком случае $A$ мохет быть унитарно преобразована в ленточную матрицу c шириной ленты, не превосходящей $4 k+3$, посредством конечного прочесса, использующего лищь арифметические операции и квадратные корни.

ДокАЗАТЕЛЬСТво. Напомним, что число $w_{i}$ имеет смысл приращения размерности обобщенного крыловского подпространства $L_{i}$ по сравнению с $L_{i-1}$. Обозначим через $r_{i}\left(s_{i}\right)$ составляющую этого приращения, полученную за счет векторов $i$-го слоя последовательности (17), в выражениях которых первьп слева множителем является матрица $B$ (соответственно $C$ ). Очевидно,

$$
w_{i}=r_{i}+s_{i}
$$

Пусть $m$ - максимальное среди значений индекса $i$ таких, что $s_{i} \neq 0$. Тогда

$$
\sum_{i=1}^{m} s_{i} \leqslant k .
$$

Слой $i$ последовательности (17) может быть получен умножением матрищы $B$, а затем $C$ на все векторы $(i-1)$-го слоя, отсюда

$$
r_{i} \leqslant w_{i-1}=r_{i-1}+s_{i-1} .
$$

Используя соотношения (18)-(20), находим

$$
w_{m}=r_{m}+s_{m} \leqslant r_{m-1}+s_{m-1}+s_{m} \leqslant \cdots \leqslant r_{1}+s_{1}+\cdots+s_{m} \leqslant r_{1}+k \leqslant 1+k .
$$


Поскольку $s_{i}=0$ при $i>m$, имеем

$$
r_{i}=w_{i} \leqslant w_{i-1} \leqslant \cdots \leqslant w_{m} \leqslant 1+k, \quad i>m
$$

Итак, $w_{i} \leqslant 1+k$ для всех $i$, т.е. в неравенстве (5) можно положить $b=1+k$, откуда и следует нужное утверждение.

Теперь мы рассмотрим обобщение теоремы 8 в другом направлении: одноранговое (не обязательно косоэрмитово) возмушение $R$ будет придаваться (вместо эрмитовой) произвольной нормальной матрице $N$. Положим

$$
A=N+R
$$

и запишем $R$ в форме внешнего произведения

$$
R=x y^{*}, \quad x, y \in \mathbb{C}^{n} .
$$

Векторы $x$ и $y$, в общем случае, линейно независимы.

Выберем произвольно ненулевой вектор $v$ и определим последовательность линейных подпространств $\left\{M_{i}\right\}$ формулами

$$
\begin{gathered}
M_{1}=\operatorname{span}\left\{x, y, v, N v, N^{*} v\right\}, \\
M_{i+1}=\operatorname{span}\left\{M_{i}, N M_{i}, N^{*} M_{i}\right\}, \quad i=1,2, \ldots .
\end{gathered}
$$

Правую часть равенства (24) нужно понимать как линейную оболочку столбцов указанных трех матриц.

Применяя матрицу $N$ к начальным векторам $v, x$ и $y$, построим три обобщенных крыловских последовательности; соответствующие крыловские подпространства обозначим через $L_{i}, \widetilde{L}_{i}$ и $\widehat{L}_{i}$.

Лемма 1. $L_{i} \subset M_{i} \subset \operatorname{span}\left\{L_{i}, \widetilde{L}_{i-1}, \widehat{L}_{i-1}\right\}, i=1,2, \ldots$.

ДокаЗАтЕльство. Если учесть, что $L_{1}=\operatorname{span}\left\{v, N v, N^{*} v\right\}, \widetilde{L}_{0}=\operatorname{span}\{x\}, \widehat{L}_{0}=$ $\operatorname{span}\{y\}$, то утверждение леммы при $i=1$ следует из формулы (23). Для $i \geqslant 2$ лемма легко доказьвается по индукции. При этом используется то, что подпространства $M_{i}$ при $i \geqslant 2$ генерируются так же, как обобщенные подпространства Крылова $L_{i}$.

Пусть $w_{i}(i=0,1,2, \ldots)$ есть ширина $i$-го слоя в матричной последовательности

$$
I, N, N^{*}, N^{2}, N N^{*}, N^{* 2}, N^{3}, N^{2} N^{*}, N N^{* 2}, N^{* 3}, \ldots
$$

Введем, кроме того, числа

$$
\bar{l}_{i}=\operatorname{dim} M_{i}, \quad i=1,2, \ldots, \quad \bar{w}_{1}=\bar{l}_{1}, \quad \bar{w}_{i}=\bar{l}_{i}-\bar{l}_{i-1}, \quad i=2,3, \ldots
$$

ЛЕМма 2. Справедливы неравенства

$$
\bar{w}_{1} \leqslant 3+w_{1}, \quad \bar{w}_{i} \leqslant w_{i}+2 w_{i-1}, \quad i=2,3, \ldots
$$

Эти неравенства непосредственно вытекают из леммы 1 и формул (23), (24). 


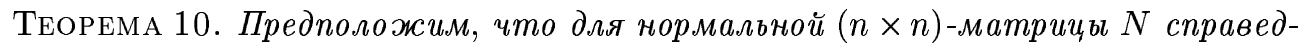
лива равномерная оченка ширины слоев $w_{i} \leqslant b, i=1,2, \ldots$, причем $2 \leqslant b<n / 6$. Тогда всякая матрица $A$ вида (21), где $R$ - одноранговое возмущение (22), может быть унитарно преобразована в ленточную матрицу с иириной ленты, не превосходящей $12 b-1$, посредством конечного процесса, использующего лишь арифметические операции и квадратные корни.

ДокАЗАТЕЛьство. Применение обобщенного процесса Ланщоша к последовательности подпространств $\left\{M_{i}\right\}$ порождает ленточную матрицу, унитарно подобную матрице $A$. Подсчет ширины ленты производится так же, как в п. 2 (см. замечание 2). Нужно лишь вместо чисел $w_{i}$ использовать числа $\bar{w}_{i}$. Согласно лемме 2 и условию теоремы $\bar{w}_{i} \leqslant 3 b, i=1,2, \ldots$.

В связи с теоремой 10 упомянем о недавнем результате М. Крупника [11]: всякую нормальную матрицу $N$ с простым спектром можно превратить в нильпотентную матрицу $A$ посредством некоторого однорангового возмущения $R$. Согласно теореме такие нильпотенты $A$ унитарно подобны ленточньм матрицам.

\section{СПИСОК ЦИТИРОВАННОЙ ЛИТЕРАТУРЫ}

[1] Bunse-Gerstner A., Elsner L. Schur parameter pencils for the solution of the unitary eigenproblem // Linear Algebra Appl. 1991. V. 154-156. P. 741-778.

[2] Elsner L., Ikramov Kh. D. On a condensed form for normal matrices under finite sequences of elementary unitary similarities // Linear Algebra Appl. 1997. V. 254. P. 79-98.

[3] Longstaff W. E. On tridiagonalization of matrices // Linear Algebra Appl. 1988. V. 109. P. 153-163.

[4] Sturmfels B. Tridiagonalization of complex matrices and a problem of Longstaff // Linear Algebra Appl. 1988. V. 109. P. 165-166.

[5] Fong C. K., Wu P. Y. Band-diagonal operators // Linear Algebra Appl. 1996. V. 248. P. 185-204.

[6] Икрамов Х. Д. Каноническая форма Шура унитарно квазидиагонализуемой матрицы // ЖВМиМФ. 1997. Т. 37. №12. С. 1411-1415.

[7] Gaines F. J., Laffey T.J., Shapiro H.M. Pairs of matrices with quadratic minimal polynomials // Linear Algebra Appl. 1983. V. 52-53. P. 289-292.

[8] Kreyszig E. Ein Satz über Matrixeigenwerte // Elem. Math. 1970. V. 25. № 3. P. 60-61.

[9] Hoffman A. J., Taussky O. A characterization of normal matrices // J. Res. Nat. Bur. Standards. 1954. V. 52. P. 17-19.

[10] Hartwig R.E., Spindelboeck K. Matrices for which $A^{*}$ and $A^{+}$commute // Linear and Multilinear Algebra. 1984. V. 14. P. 241-256.

[11] Krupnik M. Changing the spectrum of an operator by perturbation // Linear Algebra Appl. 1992. V. 167. P. 113-118. 